NASA

Technical Memorandum 100255
AVSCOM

Technical, Report 87-C-4

\title{
Finite-Element Grid Improvement by Minimization of Stiffness Matrix Trace
}

Madan G. Kittur and Ronald L. Huston

University of Cincinnati

Cincinnati, Ohio

and

Fred B. Oswald

U.S. Army Aviation Research and Technology Activity-AVSCOM

Lewis Research Center

Cleveland, Ohio

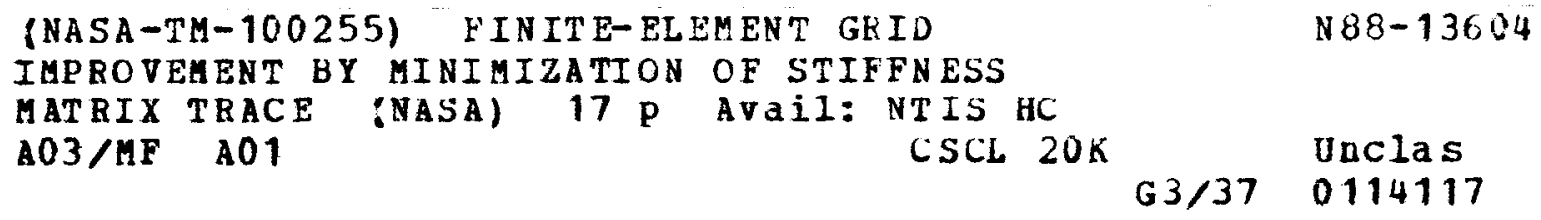

December 1987

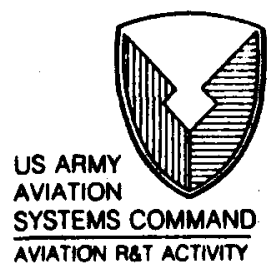


FINITE-ELEMENT GRID IMPROVEMENT BY MINIMIZATION OF STIFFNESS MATRIX TRACE

\author{
Madan G. Kittur and Ronald L. Huston \\ Department of Mechanical and \\ Industrial Engineering \\ University of Cincinnati \\ Cincinnati, Ohio 45221-0072 \\ and \\ Fred B. Oswald \\ National Aeronautics and Space Administration \\ Lewis Research Center \\ Cleveland, Ohio 44135-3191
}

\title{
SUMMARY
}

A new and simple method of finite-element grid improvement is presented. The objective is to improve the accuracy of the analysis. The procedure is based on a minimization of the trace of the stiffness matrix. For a broad class of problems this minimization is seen to be equivalent to minimizing the potential energy. The method is illustrated with the classical tapered bar problem examined earlier by Prager and by Masur. Identical results are obtained.

\section{INTRODUCTION}

In a general context, the finite-element method is an approximate procedure for solving differential equations. The accuracy of the method depends on (1) the number of elements, (2) the choice of interpolation functions, and (3) the location of the grid points. The number of elements, and hence the number of grid points, is usually restricted simply by computer capability and by processing costs. Also, there have been many recent advances in improving the accuracy of the finite-element method by using higher order interpolation polynomials and shape functions ( $p$-method) and by exhaustive analysis with large numbers of elements ( $h$-method). Some of these advances are described in the references cited herein. However, optimal grid point location ( $r$-method) is far less advanced. Practical procedures for the analyst still need to be developed and refined.

One of the earliest attempts to develop a grid optimization procedure was that of Prager in 1975 (ref. 1). Prager's work provided a stimulus and a basis for later grid optimization research as recorded in references 2 to 19 . Noteworthy among these efforts are the works of Shepard (refs. 11, 13, and 15), Masur (ref. 2), Turcke (refs. 8 and 9), Carroll (ref. 7), NcNiece (ref. 4), Carey (ref. 16), Diaz (ref. 12), Melosh (ref. 10), Durocher (ref. 17), and their colleagues.

Prager examined a bar with a linearly varying cross section under tension. He showed that the grid producing the desired least potential energy is the one where the cross-section areas at the nodes form a geometric series. In this configuration, the strain energy is divided equally among the elements. 
Masur (ref. 2) observes that this latter result of equal element strain energies is not a general characteristic of optimal meshes but instead is a result of the simple geometry of Prager's problem.

In this paper we present a finite-element grid improvement technique which is based on the minimization of the trace of the global stiffness matrix. We show that this method leads to identical results to those of Prager. It has the advantage of being simpler than traditional optimization procedures.

The method presented herein provides a mesh improvement which is based on the geometry of the body. As such, it provides a significant improvement over uniform meshes, and it produces a good first iteration for accommodating special loading configurations.

In the usual finite-element procedure, the governing equations are obtained by minimizing a functional $\pi$ by varying the dependent variables of the physical problem (ref. 20). For elastostatics this is equivalent to the principle of minimum potential energy (ref. 21). This leads to the familiar system of linear algebraic equations. Attempts to minimize $\pi$ with respect to the nodal coordinates, however, leads to a system of nonlinear equations. These equations are generally extremely difficult to solve even for the simplest cases. To avoid this difficulty we are proposing instead to examine the stiffness matrix to obtain information about an improvement in grid point location. Our motivation is the observation of the major role of the stiffness matrix in the value of the potential $\pi$. Also, the entries of the stiffness matrix are dependent on the grid point coordinates.

\section{NOMENCLATURE}
A area
Ak nodal area
$A_{\ell} \quad$ end area
Ao base area
$\bar{A}_{k}$ area of $k^{\text {th }}$ element
c area ratio (see eq. (7))
E elastic modulus
(f) global force array
\{f\} transformed global force array
$\hat{f}_{j}$ entries of $\{\hat{f}\}$
[K] global stiffness matrix
$[\hat{K}]$ diagonal form of $[\hat{K}]$ 


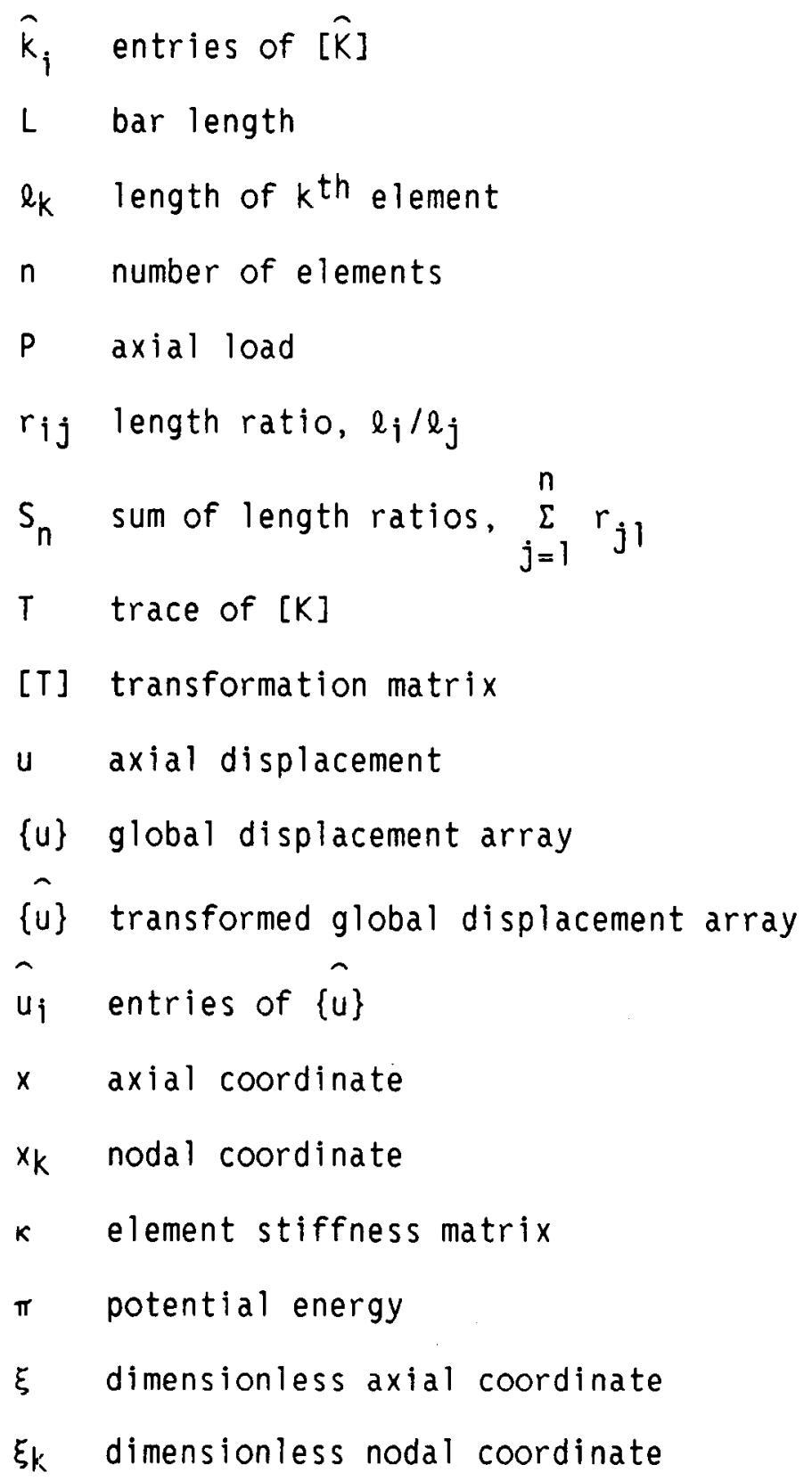

\{u\} global displacement array

\{u\} transformed global displacement array

\section{ANALYSIS}

Our objective is to develop a practical and efficient procedure of grid enhancement tending towards optimization. Our thesis is that for many problems the minimization of the trace of the stiffness matrix leads to a minimization of the potential energy and, as a consequence, provides the optimal grid configuration.

To see this, consider the governing matrix equation of finite-element analysis:

$$
[K]\{u\}=\{f\}
$$

where $[K]$ is the stiffness matrix, $\{u\}$ the array of dependent variables, and $\{f\}$ the force array. We can view [K] as an operator which maps $\{u\}$ into 
$\{f\}$. In this context, since $[K]$ is symmetricl we can find an orthogonal transformation [T] which diagonalizes [K]; that is,

$$
[\hat{K}]=[T]^{\top}[K][T]
$$

where $[\hat{K}]$ is a diagonal matrix. Let $[T]\{u\}$ and $[T]\{f\}$ be $\hat{\{u}\}$ and $\{\hat{f}\}$. Then the potential energy $\pi$ may be expressed as

$$
\pi=\frac{1}{2}\{u\}^{\top}[K]\{u\}-\{f\}^{\top}\{u\}=\frac{1}{2}\{\hat{u}\}^{\top}[\hat{K}]\{\hat{u}\}-\{\hat{f}\}^{\top}\{\hat{u}\}
$$

In terms of the array components, $\pi$ becomes

$$
\pi=\sum_{i=1}^{n}\left(\frac{1}{2} \hat{k}_{i} \hat{u}_{i}^{2}-\hat{f}_{i} \hat{u}_{i}\right)
$$

where the $\hat{k}_{j} \quad(i=1, \ldots, n)$ are the diagonal entries of $[\hat{K}]$.

Observe in equation (4) that the last term $-\sum_{i=1}^{n} \hat{f}_{i} \hat{u}_{i}$ does not explicitly involve the nodal coordinates. Therefore, $-\sum_{i=1}^{n} \hat{f}_{i} \hat{u}_{i}$ does not affect the minimization of $\pi$ with respect to the nodal coordinates. Also, since the $\hat{u}_{1}^{2}$ are positive and are independent variables in the minimization of $\pi$, the minimization of $\pi$ with respect to the nodal coordinates occurs when the sum of the $\hat{k}_{i}$ (the trace of $[\hat{K}]$ ) is a minimum. Since the trace of a matrix is invariant under an orthogonal transformation, minimizing the trace of $[\hat{K}]$ is equivalent to minimizing the trace of [K].

In minimizing the trace, we will not adversely affect the diagonal dominance of $[K]$ required to avoid ill-conditioning. The improved stiffness matrix we seek is the result of redistribution of the nodes and not of an arbitrary mathematical operation.

To illustrate the application of these concepts, consider the axially loaded tapered bar shown in figure 1. (This is the same problem examined by Prager (ref. 1) and Masur (ref. 2).) The objective is to determine a finiteelement mesh which best predicts the axial displacement. Let the bar have length $L$ and let it be divided into $n$ elements with $n+1$ nodes (numbered 0 to $n$ ) as shown. Let the areas at the ends of the bar be $A_{0}$ and $A_{l}$. Let $\xi$ be the nondimensional length parameter defined as

$$
\xi=\frac{X}{L}
$$

The analysis which follows is based on the symmetry of [K]. If [K] is not symmetric, a similar analysis could be developed using nonorthogonal transformations. 
Then the area at any particular $\xi$ along the bar is

$$
A=A_{O}(1-c \xi)
$$

where $c$ is

$$
c=\frac{A_{0}-A_{l}}{A_{0}} \quad 0 \leq c \leq 1
$$

Hence, the area at the $k^{\text {th }}$ node is

$$
A_{k}=A_{0}\left(1-c \xi_{k}\right)
$$

where $\xi_{k}$ is $\xi\left(x_{k}\right)$.

Let the individual elements have a uniform_cross section. For example, let the $k^{\text {th }}$ element have cross-section area $\bar{A}_{k}$ and length $\ell_{k}$ as in figure 2. (Note that the elements do not necessarily have the same length.) Then $\bar{A}_{k}$ and $\ell_{k}$ are

and

$$
\bar{A}_{k}=\frac{A_{k-1}+A_{k}}{2}
$$

$$
\left.e_{k}=x_{k}-x_{k-1}=L\left(\xi_{k}-\xi_{k-1}\right)\right\}
$$

The element stiffness matrix for the $k^{\text {th }}$ element is (ref. 20)

$$
[K]=\frac{\bar{A}_{k} E}{l_{k}}\left[\begin{array}{cc}
1 & -1 \\
-1 & 1
\end{array}\right]
$$

where $E$ is the elastic modulus. Then the trace $T$ of the global stiffness matrix is

$$
T=2 E \sum_{i=1}^{n} \frac{\bar{A}_{i}}{l_{i}}=\frac{E}{L} \sum_{i=1}^{n}\left(\frac{A_{i-1}+A_{i}}{\xi_{i}-\xi_{i-1}}\right)
$$

The improved node location occurs when the trace is minimized with respect to the nodal coordinates $\xi_{k}(k=1, \ldots, n-1)$. 2 Hence, by setting the derivative of $T$ with respect to $\xi_{k}$ equal to zero, we obtain

2 Nodes numbers 0 and $n$ are fixed at the ends of the bar and thus not considered for optimization. 


$$
\frac{\partial T}{\partial \xi_{k}}=0=\frac{E}{L}\left[\frac{\frac{\partial}{\partial \xi_{k}}\left(A_{k-1}+A_{k}\right)}{\xi_{k}-\xi_{k-1}}+\frac{\frac{\partial}{\partial \xi_{k}}\left(A_{k}+A_{k+1}\right)}{\xi_{k+1}-\xi_{k}}-\frac{A_{k-1}+A_{k}}{\left(\xi_{k}-\xi_{k-1}\right)^{2}}+\frac{A_{k}+A_{k+1}}{\left(\xi_{k+1}-\xi_{k}\right)^{2}}\right]
$$

Using equation (9) and simplifying we obtain

$$
A_{k+1}=A_{k}\left[\left(\frac{l_{k+1}}{\ell_{k}}\right)^{2}-1\right]+A_{k-1}\left(\frac{l_{k+1}}{\ell_{k}}\right)^{2}+c A_{0}\left(\frac{\ell_{k+1}}{L}\right)\left[\left(\frac{\ell_{k+1}}{\ell_{k}}\right)+1\right]
$$

To simplify the analysis it is convenient to introduce the length ratio parameter $r_{i j}$ defined as $\ell_{j} / \ell_{j}$. Then the ratio $\ell_{k+1} / \ell_{k}$ may be written as

$$
\frac{\ell_{k+1}}{\ell_{k}}=\frac{\frac{\ell_{k+1}}{\ell_{1}}}{\frac{\ell_{k}}{\ell_{1}}}=\frac{r_{k+1,1}}{r_{k 1}}
$$

Then $L / l_{k}$ is

$$
\frac{L}{\ell_{k}}=\sum_{j=1}^{n} \frac{\ell_{j}}{\ell_{k}}=\sum_{j=1}^{n} \frac{r_{j l}}{r_{k 1}}=\frac{s_{n}}{r_{k 1}}
$$

where $S_{n}$ is defined as $\sum_{j=1}^{n} r_{j l}$. Using this notation, we can rewrite equation (13) as

$$
A_{k+1}=A_{k}\left[\left(\frac{r_{k+1,1}}{r_{k 1}}\right)^{2}-1\right]+A_{k-1}\left(\frac{r_{k+1,1}}{r_{k 1}}\right)^{2}+\frac{c A_{0} r_{k+1,1}}{S_{n}}\left[\left(\frac{r_{k+1,1}}{r_{k 1}}\right)+1\right]
$$

Also, $\xi_{k}$ may be written as

$$
\xi_{k}=\frac{\ell_{1}+\ell_{2}+\ldots+\ell_{k}}{L}=\frac{1+r_{21}+r_{31}+\cdots+r_{k 1}}{S_{n}}=\frac{1}{S_{n}} \sum_{j=1}^{n} r_{j 1}=\frac{S_{k}}{S_{n}}
$$


Then, from equation (8), $A_{k}$ may be written as

$$
A_{k}=A_{0}\left[1-c\left(\frac{S_{k}}{S_{n}}\right)\right]
$$

Specifically, $A_{1}$ and $A_{2}$ are

$$
A_{1}=A_{0}\left[1-\left(\frac{c}{S_{n}}\right)\right]
$$

and

$$
\left.A_{2}=A_{0}\left[1-c \frac{\left(1+r_{21}\right)}{S_{n}}\right]\right\}
$$

To obtain the element area ratios let $k=1$ in equation (16). $A_{2}$ is then

$$
A_{2}=A_{1}\left(r_{21}^{2}-1\right)+A_{0} r_{21}^{2}+C A_{0} r_{21} \frac{r_{21}+1}{S_{n}}
$$

Then, by using equations (19) to eliminate $A_{1}$ and $A_{2}$, we obtain

$$
S_{n}\left(1-r_{21}\right)=c
$$

From the first of equations (19) we have

$$
A_{1}=A_{0} r_{21}
$$

or

$$
\frac{A_{1}}{A_{0}}=r_{21}
$$

Similarly, the second of equations (19) leads to

$$
A_{2}=A_{o} r_{21}^{2}
$$

or

$$
\frac{A_{2}}{A_{1}}=r_{21}
$$


Next, let $k=2$ in equation (16). By using the same procedure we obtain

$$
1-r_{32}=\frac{c}{s_{n}}\left(1-r_{32}+r_{21}\right)
$$

But, in view of equation (21) this becomes

or

$$
1-r_{32}=\left(1-r_{21}\right)\left(1-r_{32}+r_{21}\right)
$$

$$
r_{21}\left(r_{32}-r_{21}\right)=0
$$

Thus,

$$
r_{32}=r_{21}
$$

From equation (18) we have

$$
A_{3}=A_{0}\left[1-c\left(\frac{S_{3}}{S_{n}}\right)\right]=A_{0} r_{21}^{3}
$$

Therefore, we obtain

$$
\frac{A_{3}}{A_{2}}=\frac{A_{2}}{A_{1}}=\frac{A_{1}}{A_{0}}=r_{21}=r_{32}
$$

Proceeding similarly for $k=3,4, \ldots$, we obtain

$$
\frac{A_{n}}{A_{n-1}}=\frac{A_{n-1}}{A_{n-2}}=\ldots=\frac{A_{1}}{A_{0}}=r_{21}=r_{32}=\ldots=r_{n, n-1}
$$

Thus, we have the relations

$$
\begin{gathered}
r_{31}=r_{21} r_{32}=r_{21}^{2} \\
r_{41}=r_{43} r_{32} r_{21}=r_{21}^{3}, \ldots, r_{k 1}=r_{21}^{k-1}
\end{gathered}
$$

Hence, $S_{k}$ is the geometric series

$$
s_{k}=1+r_{21}+r_{21}^{2}+\ldots+r_{21}^{k-1}=\frac{1-r_{21}^{k}}{\left(1-r_{21}\right)}
$$


Then, from equations (18) and (21), we have

$$
A_{k}=A_{o}\left[1-\left(1-r_{21}^{k}\right)\right]=A_{o} r_{21}^{k}
$$

and then

$$
A_{n}=A_{0} r_{21}=A_{\ell}
$$

Then, from equation (7) we see that $r_{21}$ is

$$
r_{21}=(1-c)^{1 / n}
$$

Finally, by substituting into equation (17) we have

$$
\begin{gathered}
\xi_{k}=\frac{\ell_{1}}{L}\left(1+r_{21}+r_{31}+\ldots+r_{k 1}\right)=\left(1-r_{21}\right) \frac{1+r_{21}+r_{21}^{2}+\ldots+r_{21}^{k-1}}{c} \\
=\frac{1-r_{21}^{k}}{c}=\frac{1-(1-c)^{k / n}}{c}
\end{gathered}
$$
problem.

This is the result obtained by Prager (ref. 1) in his analysis of the same

\section{DISCUSSION}

First, observe that in equation (34) for a uniform thickness beam $c=0$ and thus $\xi_{k}$ is undetermined. This means that for a uniform thickness beam the nodal positions are arbitrary; that is, all meshes are equally optimal for a uniform thickness beam.

Next, consider again the element stiffness matrix of equation (10). From equations (8) and (34) the scalar multiplier is

$$
\frac{E \bar{A}_{k}}{\ell_{k}}=\frac{E\left(A_{k-1}+A_{k}\right)}{2 L\left(\xi_{k}-\xi_{k-1}\right)}=\frac{A_{o} c}{2 L}\left(\frac{1+r_{21}}{1-r_{21}}\right)
$$

Since this is a constant (independent of $k$ ) the element stiffness matrix is the same for each element. This means that each element has the same strain energy. Masur (ref. 2) has suggested that this result is due to the simple geometry of the problem.

Even with this simple geometry, however, the analysis needed to determine the optimal nodal positions has been extremely detailed. With more complex geometries the analysis will become intractable. Alternatively, a more convenient method of improving the nodal positions is to examine the trace of the stiffness matrix and to adjust the nodal positions to minimize the trace. The criteria for minimizing the trace of the stiffness matrix is a comparatively 
simple procedure - readily amenable to the development of computer algorithms for optimal nodal locations.

In the preceeding discussion, we have ignored any consideration of the effects of concentrated loads or boundary/edge effects. It is common engineering practice to use a fine (dense) grid in highly loaded regions and to place a grid point specifically at the point of application of a concentrated load. The trace minimization suggested here is intended to aid in the discretization process at locations removed from concentrated loads. Our justification is based on Saint-Venant's principle that localized effects disappear at short distances.

A principal question with the minimum trace method is: What is the range of applicability? The authors have found the method to lead to significant decreases in potential energy from that of a uniform mesh for structural and heat-transfer problems. The range of applicability is currently being explored. Numerical algorithms using this procedure are being developed. Finally, the influence of values of second and higher invariants of the stiffness matrix needs to be explored.

\section{NUMERICAL EXAMPLE}

To illustrate the value of optimizing the mesh, consider an axially loaded bar which tapers to $1 / 3$ the base area as in figure 3 . Specifically, let $P$, $A_{O}, C, E$, and $L$ have the following values:

$$
\begin{gathered}
P=20 \mathrm{~N} \\
A_{0}=0.0015 \mathrm{~m}^{2} \\
C=\frac{2}{3} \\
E=2.07 \times 10^{11 \mathrm{~N} / \mathrm{m}^{2}} \\
L=4 \mathrm{~m}
\end{gathered}
$$

The objective is to find the axial displacement. is

From elementary mechanics the axial displacement $u$ at any location $x$

$$
u=-\frac{P L}{A_{0} E C} \ln \left(1-\frac{C X}{L}\right)
$$

To compare the displacement results of finite-element models with equation (37), four models of the bar, each having four elements, were examined. One of the models had a uniform nodal distribution. Another had the "optimal" mesh as developed in equation (34). The remaining two models had arbitarily selected nodal distributions. The nodal displacements were evaluated using the four models and compared with the displacement calculated by equation (37). Table I shows the results. Table II presents an error analysis and also an 
$L_{2}$-norm of the error. As expected, the optimum mesh produces the least $L_{2}$ error.

\section{CONCLUSIONS}

A new method of finite-element grid improvement based on the principle of minimization of the trace of the stiffness matrix was developed. This procedure is equivalent to minimizing the potential energy of the model by dividing the strain energy equally among the elements. The following conclusions are made:

1. The analysis and the numerical results demonstrate the potential usefulness of the trace minimization mesh improvement method.

2. Minimization of the trace of the stiffness matrix is a relatively simple mesh optimization procedure. It is readily adaptable to algorithm development.

3. Trace minimization can be used in combination with other grid optimization techniques. Indeed, it can provide a starting mesh for iteration techniques, useful for specialized loading.

4. The principal benefit of the trace minimization method is accuracy of analysis as opposed to efficiency of analysis.

\section{REFERENCES}

1. Prager, W.: A Note on the Optimal Choice of Finite Element Grids. Comput. Methods Appl. Mech. Eng., vol. 6, no. 3, Nov. 1975, pp. 363-366.

2. Masur, E.F.: Some Remarks on the Optimal Choice of Finite Element Grids. Comput. Methods Appl. Mech. Eng., vol. 14, May 1978, pp. 237-248.

3. Tang, J.W. and Turcke, D.J.: Characteristics of the Optimal Grids. Comput. Methods Appl. Mech. Eng., vol. 11, no. 1, Apr. 1977, pp. 31-37.

4. McNeice, G.M. and Marcal, P.V.: Optimization of Finite Element Grids Based on Minimum Potential Energy. J. Eng. Ind., vol. 95, no. 1, Feb. 1973, pp. $186-190$.

5. Carroll, W.E.: Inclusive Criteria for Optimum Grid Generation in the Discrete Analysis Technique. Comput. Struct., vol. 6, no. 4-5, Aug.-Oct. 1976, pp. 333-337.

6. Pederson, P.: Some Properties of Linear Strain Triangles and Optimal Finite Element Models. Int. J. Numer. Methods Eng., vol. 7, no. 4, 1973, pp. 415-431.

7. Carrol1, W.E. and Barker, R.M.: A Theorem for Optimum Finite Element Idealizations. Int. J. Solids Struct., vol. 9, no. 7, July 1973, pp. 883-895. 
8. Turcke, D.J. and McNeice, G.M.: Guidelines for Selecting Finite Element Grids Based on an Optimization Study. Comput. Struct., vol. 4, no. 3, May 1974, pp. 499-519.

9. Turcke, D.: On Optimum Finite Element Grid Configurations. AIAA J., vol. 14, no. 2, Feb. 1976, pp. 264-265.

10. Melosh, R.J. and Marcal, P.V.: An Energy Basis for Mesh Refinement of Structural Continua. Int. J. Numer. Methods Eng., vol. 11, no. 7, 1977 , pp. 1083-1092.

11. Shephard, M.S.: Finite Element Grid Optimizatiion - A Review. Finite Element Grid Optimization, M.S. Shepard and R.H. Gallagher, eds., ASME, 1979, pp. 1-13.

12. Diaz, A.R., et al.: Design of an Optimal Grid for Finite Element Methods. J. Struct. Mech., vol. 11, no. 2, 1983, pp. 215-230.

13. Shephard, M.S.; Gallagher, R.H.; and Abel, J.F.: The Synthes is of NearOptimum Finite Element Meshes with Interactive Computer Graphics. Int. J. Numer. Methods Eng., vol. 15, no. 7, 1980, pp. 1021-1039.

14. Babuska, I. and Rheinboldt, W.C.: A-Posteriori Error Estimates for the Finite Element Method. Int. J. Numer. Methods Eng., vol. 12, no. 10, 1978, pp. $1597-1615$.

15. Shephard, M.S.: An Algorithm for Defining a Single Near-Optimum Mesh for Multiple-Load-Case Problems. Int. J. Numer. Methods Eng., vol. 15, no. 4, 1980 , pp. 617-625.

16. Carey, G.F.: A Mesh-Refinement Scheme for Finite Element Computations. Comput. Methods Appl. Mech. Eng., vol. 7, no. 1, Jan. 1976, pp. 93-105.

17. Durocher, L.L. and Stango, R.J.: Finite Element Mesh Optimization and Enrichment Techniques. Struc. Mech. Software Series, Vol. III, N. Perrone, W. Pilkey, and B. Pilkey, eds., University Press of Virginia, 1980, pp. 245-262.

18. Hirai, I.; Wang, B.P.; and Pilkey, W.D.: An Efficient Zooming Method for Finite Element Analysis. Int. J. Numer. Methods Eng., vol. 20, no. 9, Sept. 1984, pp. 1671-1683.

19. Hirai, I., et al.: An Exact Zooming Method. Finite Elements Anal. Des., vol. 1, no. 1, Apr. 1985, pp. 61-69.

20. Huston, R.L. and Passerello, C.E.: Finite Element Methods - An Introduction. Marcel Dekker, 1984, pp. 28, 151.

21. Sokolnikoff, I.S.: Mathematical Theory of Elasticity. R.E. Krieger Publishing, Malabar, FL, 1983, p. 385. 
TABLE I. - COMPARISON OF AXIAL DISPLACEMENTS FOR THE BAR OF FIGURE 3 CALCULATED USING VARIOUS MODELS

\begin{tabular}{|c|c|c|c|c|c|}
\hline \multirow{2}{*}{$\begin{array}{c}\text { Axial } \\
\text { location, } \\
x \\
m\end{array}$} & \multirow{2}{*}{$\begin{array}{c}\text { Exact displacement } \\
(\text { eq. }(37)) \\
10^{-9} \mathrm{~m}\end{array}$} & \multicolumn{4}{|c|}{$\begin{array}{c}\text { Displacements computed using various models, } \\
10^{-9} \mathrm{~m}\end{array}$} \\
\hline & & $\begin{array}{l}\text { Uniform } \\
\text { mesh }\end{array}$ & $\begin{array}{l}\text { "Optimum" } \\
\text { mesh } \\
\text { (Prager) }\end{array}$ & Mesh 3 & Mesh 4 \\
\hline $\begin{array}{l}0.0 \\
.5 \\
1.0 \\
1.4409860 \\
2.0 \\
2.5 \\
2.5358986 \\
3.0 \\
3.3678522 \\
4.0\end{array}$ & $\begin{array}{c}0.0 \\
33.6276 \\
70.46244 \\
106.1461 \\
156.702 \\
208.3078 \\
212.2923 \\
267.883 \\
318.4384 \\
424.5845\end{array}$ & $\begin{array}{c}0.0 \\
70.2679 \\
156.1509 \\
266.5719 \\
421.1612\end{array}$ & $\begin{array}{c}0.0 \\
105.482 \\
210.9648 \\
316.4529 \\
421.940\end{array}$ & $\begin{array}{c}0.0 \\
33.60639 \\
70.41338 \\
206.8158 \\
\\
\\
\end{array}$ & $\begin{array}{c}0.0 \\
33.60639 \\
15.56506 \\
207.1804 \\
\\
\\
\end{array}$ \\
\hline
\end{tabular}

TABLE II. - ERROR ANALYSIS

\begin{tabular}{|c|c|c|c|c|}
\hline \multirow{2}{*}{$\begin{array}{c}\text { Axial } \\
\text { location, } \\
x, \\
m\end{array}$} & \multicolumn{4}{|c|}{$\begin{array}{l}\text { Error for various meshes, } \\
\qquad 10^{-9} \mathrm{~m}\end{array}$} \\
\hline & $\begin{array}{l}\text { Uniform } \\
\text { mesh }\end{array}$ & $\begin{array}{l}\text { "Optimum" } \\
\text { mesh } \\
\text { (Prager) }\end{array}$ & Mesh 3 & Mesh 4 \\
\hline $\begin{array}{l}0.0 \\
.5 \\
1.0 \\
1.441 \\
2.0 \\
2.5 \\
2.536 \\
3.0 \\
3.368 \\
4.0 \\
\mathrm{~L}_{2} \text {-Norm }\end{array}$ & $\begin{array}{l}0.0 \\
.1945411 \\
.5506105 \\
1.311108 \\
3.423228 \\
3.7119418\end{array}$ & $\begin{array}{l}0.0 \\
-664118 \\
1.32769 \\
1.985439 \\
2.64433 \\
3.6246009\end{array}$ & $\begin{array}{l}0.0 \\
.02121 \\
.04906 \\
\\
1.492 \\
\\
6.965 \\
7.1232118\end{array}$ & $\begin{array}{l}0.0 \\
.02121 \\
-0514 \\
1.1274 \\
6.6031 \\
6.780697\end{array}$ \\
\hline
\end{tabular}

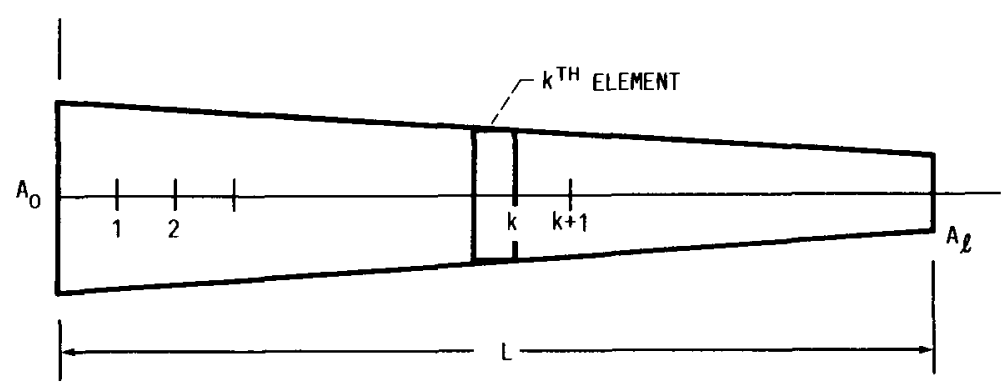

FIGURE 1. - LINEAR TAPERED BAR. 

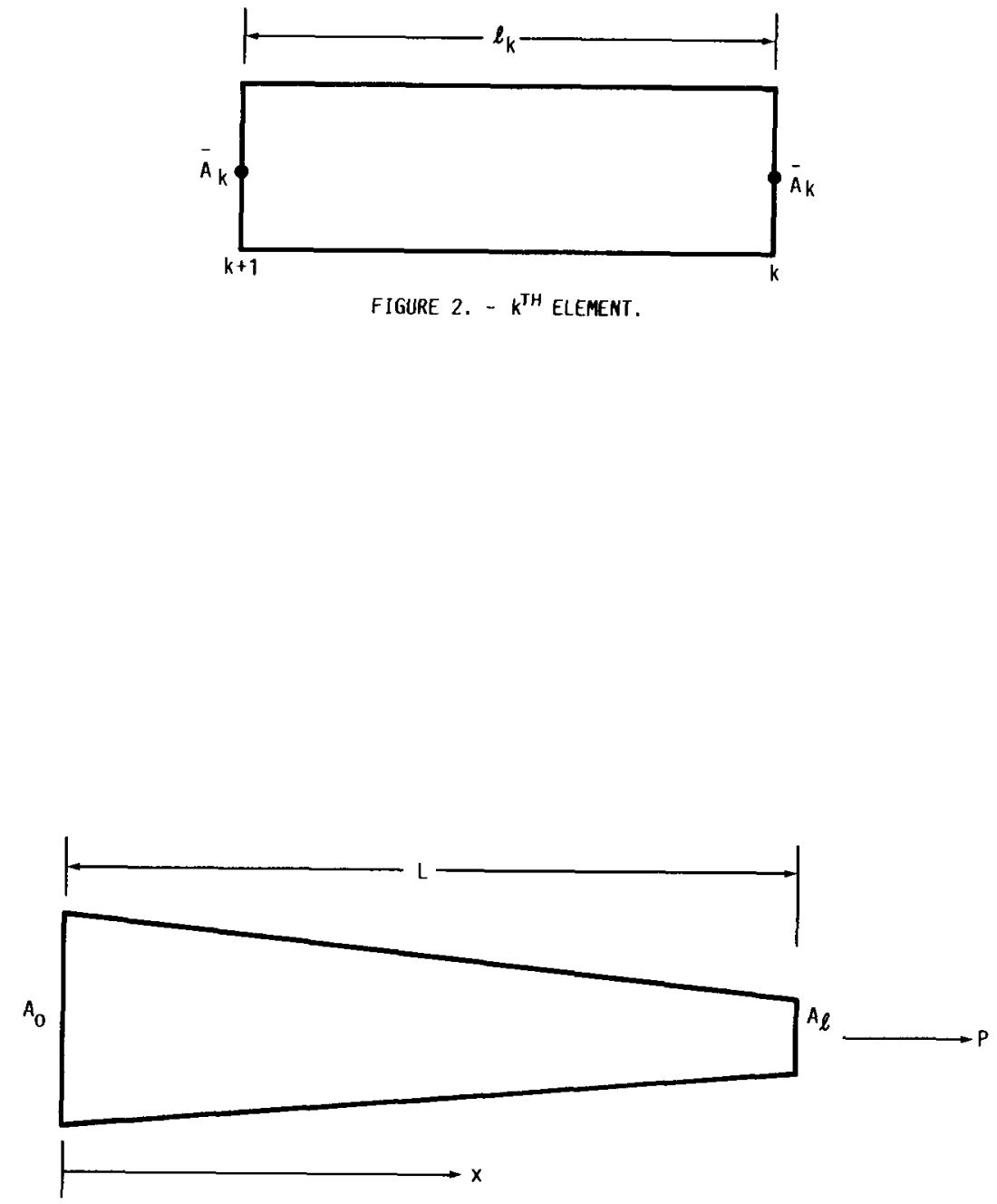

FIGURE 3. - EXAMPLE BAR AND LOADING. 


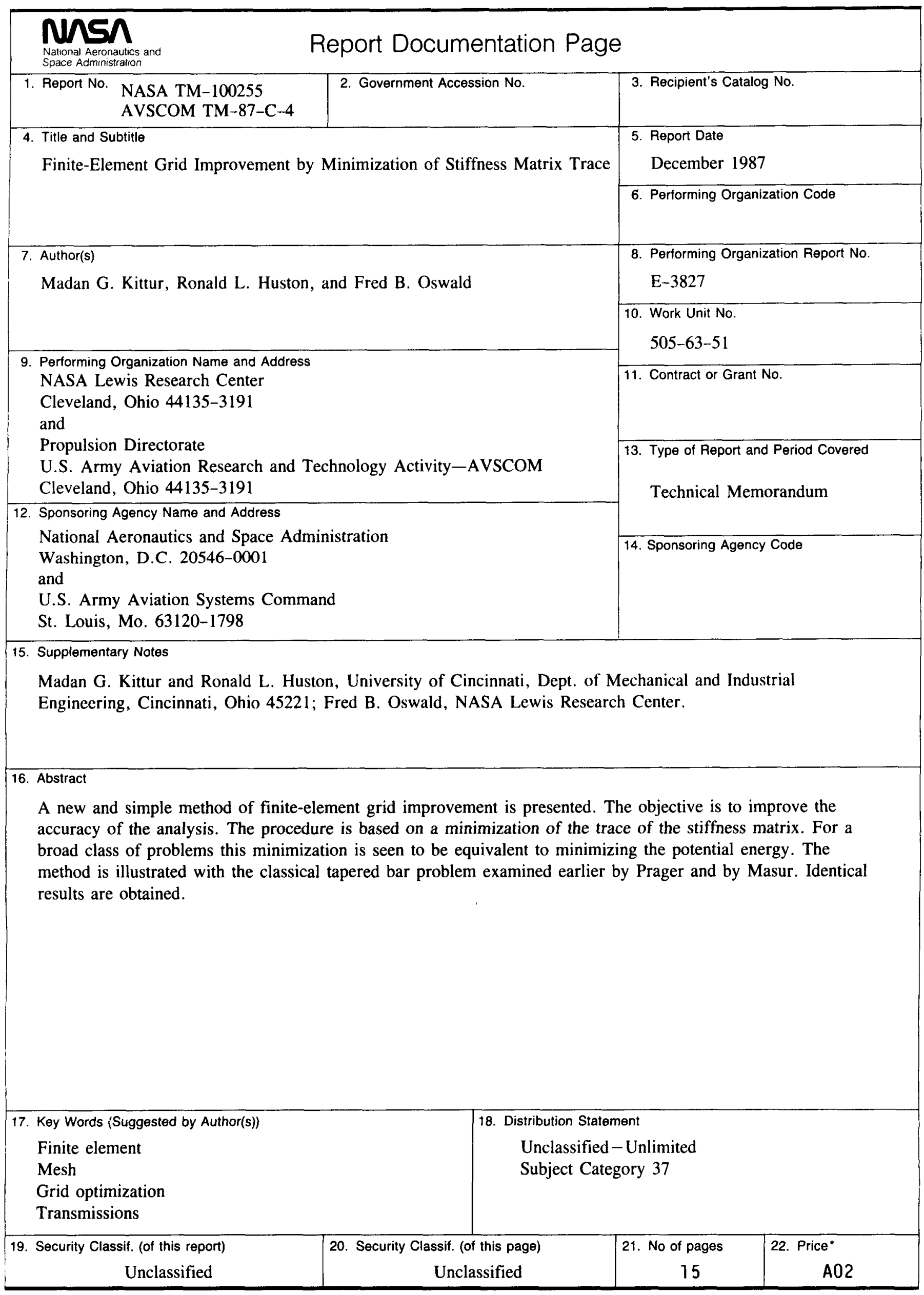

